\title{
Estimation of the parameters by using non-Bayesian in Modified Weibull Extension Distribution
}

\author{
Shaima'a B. Yass ${ }^{1}$, Iden H. Al Kanani² \\ \{shai.bader@yahoo.com ${ }^{1}$, Iden_alkanani@yahoo.com ${ }^{2}$ \} \\ Al-Iraqia University, College of Engineering, Networks Engineering Department ${ }^{1}$, \\ University of Baghdad, College of Science for Women, Department of Mathematics ${ }^{2}$
}

\begin{abstract}
In this paper, studying the modified Weibull extension model, and derive the estimators of three parameters by using maximum likelihood method, and ordinary least squares estimator method. Then by utilizing Monte Carlo method in simulation procedure for generate many various of samples sizes with many various of replicate the sample size. Finally, finding that the maximum likelihood method are the best values from the ordinary least squares method.
\end{abstract}

Keywords: Modified Weibull extension, Classical distribution methods, Newton Raphson method.

\section{Introduction}

There are number of application of Weibul distributions and modified Weibull distribution to modeling the test data in scientific research and life experiments. Also, number of research dealing with these models to estimate the parameters.

In 2002, Xie et al. [6], generalized the Weibull distribution to a modified Weibull extension, model with three parameters, and in 2005 [4], Nadarajah driving explicit algebraic formulas for the kth moment of the distribution. Furthermore, in 2012, Peng et al. [5], proposed comparative analysis to prove that the modified Weibull extension distribution is more accurate and flexible in modeling the satellite reliability than the classical Weibull distribution. The accuracy and convenience of the modified Weibull extension distribution indicates its practical application in health of living organisms and engineering designs. In 2018, Al Kanani and Kalt [3] using simulation to studied classical distribution methods of the modified Weibull distribution. In 2019, Al Kanani and Jalil [2] discussed some estimation method for new distribution (mixture distribution) to estimate the parameters. In 2021, Al Kanani and Ali [1] dealing with exponentiated Weibull as a special case of Weibul distributions for estimate the parameters. By the way, the aim of this research is estimating all the three parameters of the modified Weibull extension model, by using maximum likelihood estimator method, and ordinary least squares estimator method to show the best values of parameters.

This paper is organized as follows. In section 2 recalling the information of modified Weibull extension, such as probability density function, Cumulated distribution, the reliability function, and hazard function. Section 2, and section 3 are devoted to the parameter estimation, Maximum Likelihood Estimator, and Ordinary Least Squares Estimator, respectively. Some sample sizes are generating for the simulation procedure in section 4 , to illustrate the application of the modified Weibull extension model. Finally, comparative studies by using mean square error are used in section 5. 


\section{Modified Weibull Extension Model}

The probability density function of this model is:

$$
f(x)=\theta \gamma\left(\frac{x}{\delta}\right)^{\gamma-1} e^{\left(\frac{x}{\delta}\right)^{\gamma}+\theta \delta\left(1-e^{\left(\frac{x}{\delta}\right)^{\gamma}}\right)} ; \text { for } x, \delta, \theta, \gamma>0
$$

Where the shape parameter is $\gamma$, the scale parameters are $\delta$, and $\theta$. And, the Cumulated distribution function is:

$$
F(x)=\int_{0}^{x} f(t) d t=1-e^{\theta \delta\left(1-e^{\left(\frac{x}{\delta}\right)^{\gamma}}\right)}, x>0
$$

Where, the reliability function is:

$$
\mathrm{R}(x)=\int_{x}^{\infty} f(t) d t=e^{\theta \delta\left(1-e^{\left(\frac{x}{\delta}\right)^{\gamma}}\right)}, x>0
$$

And, the hazard function:

$$
h(x)=\frac{f(x)}{R(x)}=\theta \gamma\left(\frac{x}{\delta}\right)^{\gamma-1} e^{\left(\frac{x}{\delta}\right)} \gamma^{\gamma}, x>0
$$

\section{Maximum Likelihood Estimator Method (MLE)}

This method is one of the most widely utilizing in statistical estimation. It used to estimate the parameters of discrete and continuous distribution. The idea of this method is to find an estimate to parameter, say $\widehat{\boldsymbol{\theta}}(\boldsymbol{X})$, that maximize the likelihood (joint) function.

Taking the likelihood function of three parameters modified Weibull extension distribution to get:

$$
L\left(\delta, \theta, \gamma ; x_{i}\right)=\prod_{i=1}^{n} f\left(x_{i} ; \delta, \theta, \gamma\right)
$$

That is:

$$
L\left(\delta, \theta, \gamma ; x_{i}\right)=\theta^{n} \gamma^{n} \prod_{i=1}^{n}\left(\frac{x_{i}}{\delta}\right)^{\gamma-1} e^{\sum_{i=1}^{n}\left[\left(\frac{x_{i}}{\delta}\right)^{\gamma}+\theta \delta\left(1-e^{\left(\frac{x_{i}}{\delta}\right)^{\gamma}}\right)\right]}
$$

Taking the natural logarithm for the above function, to obtain:

$$
\operatorname{Ln} L=n \operatorname{Ln} \theta+n \operatorname{Ln} \gamma+(\gamma-1) \sum_{i=1}^{n} \operatorname{Ln}\left(\frac{x_{i}}{\delta}\right)+\sum_{i=1}^{n}\left[\left(\frac{x_{i}}{\delta}\right)^{\gamma}+\theta \delta\left(1-e^{\left(\frac{x_{i}}{\delta}\right)^{\gamma}}\right)\right]
$$

The partial derivatives for the logarithm likelihood function with respect to parameters $\delta, \theta$ and $\gamma$ are :

$$
\begin{gathered}
f_{1}(\delta)=\frac{\partial \operatorname{Ln} L}{\partial \delta} \\
f_{1}(\delta)=\frac{n}{\delta}-\frac{n \gamma}{\delta}+\sum_{i=1}^{n}\left[-\frac{\gamma}{\delta}\left(\frac{x_{i}}{\delta}\right)^{\gamma}+\theta\left(1-e^{\left(\frac{x_{i}}{\delta}\right)^{\gamma}}\right)+\theta \gamma\left(\frac{x_{i}}{\delta}\right)^{\gamma} e^{\left.\left(\frac{x_{i}}{\delta}\right)^{\gamma}\right]}\right.
\end{gathered}
$$




$$
\begin{gathered}
g_{1}(\theta)=\frac{\partial \operatorname{Ln} L}{\partial \theta}=\frac{n}{\theta}+\sum_{i=1}^{n}\left[\delta\left(1-e^{\left(\frac{x_{i}}{\delta}\right)^{\gamma}}\right)\right] \\
h_{1}(\gamma)=\frac{\partial \operatorname{Ln} L}{\partial \gamma} \\
=\frac{n}{\gamma}+\sum_{i=1}^{n} \operatorname{Ln}\left(\frac{x_{i}}{\delta}\right)+\sum_{i=1}^{n}\left[\left(\frac{x_{i}}{\delta}\right)^{\gamma} \operatorname{Ln}\left(\frac{x_{i}}{\delta}\right)-\theta \delta e^{\left(\frac{x_{i}}{\delta}\right)^{\gamma}}\left(\frac{x_{i}}{\delta}\right)^{\gamma} \operatorname{Ln}\left(\frac{x_{i}}{\delta}\right)\right]
\end{gathered}
$$

The above equations (8), (9), and (10), are system of non-linear equations, can be solved by using Newton - Raphson method. Thus we need the Jacobian matrix $J_{K 1}$, which is:

$$
J_{K 1}=\left[\begin{array}{lll}
\frac{\partial f_{1}(\delta)}{\partial \delta} & \frac{\partial f_{1}(\delta)}{\partial \theta} & \frac{\partial f_{1}(\delta)}{\partial \gamma} \\
\frac{\partial g_{1}(\theta)}{\partial \delta} & \frac{\partial g_{1}(\theta)}{\partial \theta} & \frac{\partial g_{1}(\theta)}{\partial \gamma} \\
\frac{\partial h_{1}(\gamma)}{\partial \delta} & \frac{\partial h_{1}(\gamma)}{\partial \theta} & \frac{\partial h_{1}(\gamma)}{\partial \gamma}
\end{array}\right]
$$

Where the pratial derivative are getting as follows:

$$
\begin{aligned}
& \frac{\partial f_{1}(\delta)}{\partial \delta}=\frac{\partial^{2} \operatorname{Ln} L}{\partial^{2} \delta} \\
& \frac{\partial f_{1}(\delta)}{\partial \delta}=\frac{-n}{\delta^{2}}+\frac{n \gamma}{\delta^{2}}+\frac{\gamma}{\delta^{2}} \sum_{i=1}^{n}\left(\frac{x_{i}}{\delta}\right)^{\gamma}+\frac{\gamma^{2}}{\delta^{2}} \sum_{i=1}^{n}\left(\frac{x_{i}}{\delta}\right)^{\gamma}+\frac{\theta \gamma}{\delta} \sum_{i=1}^{n} e^{\left(\frac{x_{i}}{\delta}\right)^{\gamma}}\left(\frac{x_{i}}{\delta}\right)^{\gamma} \\
& -\frac{\theta \gamma^{2}}{\delta} \sum_{i=1}^{n} e^{\left(\frac{x_{i}}{\delta}\right)^{\gamma}}\left(\frac{x_{i}}{\delta}\right)^{\gamma}-\frac{\theta \gamma^{2}}{\delta} \sum_{i=1}^{n} e^{\left(\frac{x_{i}}{\delta}\right)^{\gamma}}\left(\frac{x_{i}}{\delta}\right)^{2 \gamma} \\
& \frac{\partial f_{1}(\delta)}{\partial \theta}=\frac{\partial^{2} \operatorname{Ln} L}{\partial \theta \partial \delta} \\
& \frac{\partial f_{1}(\delta)}{\partial \theta}=n-\sum_{i=1}^{n} e^{\left(\frac{x_{i}}{\delta}\right)^{\gamma}}+\gamma \sum_{i=1}^{n} e^{\left(\frac{x_{i}}{\delta}\right)^{\gamma}}\left(\frac{x_{i}}{\delta}\right)^{\gamma} \\
& \frac{\partial f_{1}(\delta)}{\partial \gamma}=\frac{\partial^{2} \operatorname{Ln} L}{\partial \gamma \partial \delta} \\
& \frac{\partial f_{1}(\delta)}{\partial \gamma}=\frac{-n}{\delta}-\frac{1}{\delta} \sum_{i=1}^{n}\left(\frac{x_{i}}{\delta}\right)^{\gamma}-\frac{\gamma}{\delta} \sum_{i=1}^{n}\left(\frac{x_{i}}{\delta}\right)^{\gamma} \operatorname{Ln}\left(\frac{x_{i}}{\delta}\right)-\theta \sum_{i=1}^{n} e^{\left(\frac{x_{i}}{\delta}\right)^{\gamma}}\left(\frac{x_{i}}{\delta}\right)^{\gamma}
\end{aligned}
$$




$$
\begin{aligned}
& \operatorname{Ln}\left(\frac{x_{i}}{\delta}\right)+\theta \sum_{i=1}^{n} e^{\left(\frac{x_{i}}{\delta}\right)^{\gamma}}\left(\frac{x_{i}}{\delta}\right)^{\gamma}+\theta \gamma \sum_{i=1}^{n} e^{\left(\frac{x_{i}}{\delta}\right)^{\gamma}}\left(\frac{x_{i}}{\delta}\right)^{\gamma} \operatorname{Ln}\left(\frac{x_{i}}{\delta}\right)+ \\
& \theta \gamma \sum_{i=1}^{n} e^{\left(\frac{x_{i}}{\delta}\right)^{\gamma}}\left(\frac{x_{i}}{\delta}\right)^{2 \gamma} \operatorname{Ln}\left(\frac{x_{i}}{\delta}\right) \\
& \frac{\partial g_{1}(\theta)}{\partial \delta}=\frac{\partial^{2} \operatorname{Ln} L}{\partial \delta \partial \theta}=\frac{\partial f_{1}(\delta)}{\partial \theta} \\
& \frac{\partial g_{1}(\theta)}{\partial \theta}=\frac{\partial^{2} \operatorname{Ln} L}{\partial \theta^{2}}=\frac{-n}{\theta^{2}} \\
& \frac{\partial g_{1}(\theta)}{\partial \gamma}=\frac{\partial^{2} \operatorname{Ln} L}{\partial \gamma \partial \theta}=-\delta \sum_{i=1}^{n} e^{\left(\frac{x_{i}}{\delta}\right)^{\gamma}}\left(\frac{x_{i}}{\delta}\right)^{\gamma} \operatorname{Ln}\left(\frac{x_{i}}{\delta}\right) \\
& \frac{\partial h_{1}(\gamma)}{\partial \delta}=\frac{\partial^{2} \operatorname{Ln} L}{\partial \delta \partial \gamma}=\frac{\partial f_{1}(\delta)}{\partial \gamma} \\
& \frac{\partial h_{1}(\gamma)}{\partial \theta}=\frac{\partial^{2} \operatorname{Ln} L}{\partial \theta \partial \gamma}=\frac{\partial g_{1}(\theta)}{\partial \gamma} \\
& \frac{\partial h_{1}(\gamma)}{\partial \gamma}=\frac{\partial^{2} \operatorname{Ln} L}{\partial \gamma^{2}} \\
& \frac{\partial h_{1}(\gamma)}{\partial \gamma}=\frac{-n}{\gamma^{2}}+\sum_{i=1}^{n}\left(\operatorname{Ln}\left(\frac{x_{i}}{\delta}\right)\right)^{2}\left(\frac{x_{i}}{\delta}\right)^{\gamma}-\theta \delta \sum_{i=1}^{n}\left(\operatorname{Ln}\left(\frac{x_{i}}{\delta}\right)\right)^{2}\left(\frac{x_{i}}{\delta}\right)^{\gamma} e^{\left(\frac{x_{i}}{\delta}\right)^{\gamma}} \\
& -\theta \delta \sum_{i=1}^{n}\left(\operatorname{Ln}\left(\frac{x_{i}}{\delta}\right)\right)^{2}\left(\frac{x_{i}}{\delta}\right)^{2 \gamma} e^{\left(\frac{x_{i}}{\delta}\right)^{\gamma}}
\end{aligned}
$$

Thus, all above equations in section 3 , proved that the Jacobian matrix $J_{K 1}$ is non-singular symmetric matrix, therefore:

$$
\left[\begin{array}{l}
\delta_{k+1} \\
\theta_{k+1} \\
\gamma_{k+1}
\end{array}\right]=\left[\begin{array}{l}
\delta_{k} \\
\theta_{k} \\
\gamma_{k}
\end{array}\right]-J_{K 1}{ }^{-1}\left[\begin{array}{l}
f_{1}\left(\delta_{k}\right) \\
g_{1}\left(\theta_{k}\right) \\
h_{1}\left(\gamma_{k}\right)
\end{array}\right]
$$

\section{Ordinary Least Squares Estimator Method (OLS)}

This method is one of the most popular technique, to estimate the parameters when the models is linear and nonlinear in variables. The idea of this method is to minimize the sum squared differences between observed sample and the expected sample, as follows: 


$$
\sum_{i=1}^{n} \epsilon_{i}{ }^{2}=\sum_{i=1}^{n}\left[Y_{i}-E\left(Y_{i}\right)\right]^{2}
$$

Now, applying this method to minimize the sum squared difference between the estimate of cumulative distribution function, and the empirical cumulative distribution function, thus:

$$
\sum_{i=1}^{n} \epsilon_{i}{ }^{2}=\sum_{i=1}^{n}\left[F\left(x_{i}\right)-\hat{F}\left(x_{i}\right)\right]^{2}
$$

Where empirical cumulative distribution function is:

$$
F\left(x_{i}\right)=\frac{i-0.5}{n}
$$

Thus:

$$
\sum_{i=1}^{n} \epsilon_{i}{ }^{2}=\sum_{i=1}^{n}\left[\frac{n-i+0.5}{n}-e^{\theta \delta\left(1-e^{\left(\frac{x_{i}}{\delta}\right)^{\gamma}}\right)}\right]^{2}
$$

The partial derivatives for the logarithm likelihood function with respect to parameters $\delta, \theta$ and $\gamma$ are :

$$
\begin{aligned}
& f_{2}(\delta)=\frac{\partial \sum_{i=1}^{n} \epsilon_{i}^{2}}{\partial \delta} \\
& f_{2}(\delta)=-2 \theta \sum_{i=1}^{n}\left[\frac{n-i+0.5}{n} e^{\theta \delta\left(1-e^{\left(\frac{x_{i}}{\delta}\right)^{\gamma}}\right)}-e^{2 \theta \delta\left(1-e^{\left(\frac{x_{i}}{\delta}\right)^{\gamma}}\right)}\right]\left[1-e^{\left(\frac{x_{i}}{\delta}\right)^{\gamma}}+\right. \\
& \left.\gamma e^{\left(\frac{x_{i}}{\delta}\right)^{\gamma}}\left(\frac{x_{i}}{\delta}\right)^{\gamma}\right] \\
& g_{2}(\theta)=\frac{\partial \sum_{i=1}^{n} \epsilon_{i}^{2}}{\partial \theta} \\
& g_{2}(\theta)=-2 \delta \sum_{i=1}^{n}\left[\frac{n-i+0.5}{n} e^{\theta \delta\left(1-e^{\left(\frac{x_{i}}{\delta}\right)^{\gamma}}\right)}-e^{2 \theta \delta\left(1-e^{\left(\frac{x_{i}}{\delta}\right)^{\gamma}}\right)}\right]\left(1-e^{\left(\frac{x_{i}}{\delta}\right)^{\gamma}}\right) \\
& h_{2}(\gamma)=\frac{\partial \sum_{i=1}^{n} \epsilon_{i}^{2}}{\partial \gamma} \\
& h_{2}(\gamma)=2 \theta \delta \sum_{i=1}^{n}\left[\frac{n-i+0.5}{n} e^{\theta \delta\left(1-e^{\left(\frac{x_{i}}{\delta}\right)^{\gamma}}\right)+\left(\frac{x_{i}}{\delta}\right)^{\gamma}}-e^{2 \theta \delta\left(1-e^{\left(\frac{x_{i}}{\delta}\right)^{\gamma}}\right)+\left(\frac{x_{i}}{\delta}\right)^{\gamma}}\right] \\
& \left(\frac{x_{i}}{\delta}\right)^{\gamma} \operatorname{Ln}\left(\frac{x_{i}}{\delta}\right)
\end{aligned}
$$


The above equations (24), (25), and (26) are system of non-linear equations, can be solved by using Newton - Raphson method. Thus we need the Jacobian matrix $J_{K 2}$, which is:

$$
J_{K 2}=\left[\begin{array}{lll}
\frac{\partial f_{2}(\delta)}{\partial \delta} & \frac{\partial f_{2}(\delta)}{\partial \theta} & \frac{\partial f_{2}(\delta)}{\partial \gamma} \\
\frac{\partial g_{2}(\theta)}{\partial \delta} & \frac{\partial g_{2}(\theta)}{\partial \theta} & \frac{\partial g_{2}(\theta)}{\partial \gamma} \\
\frac{\partial h_{2}(\gamma)}{\partial \delta} & \frac{\partial h_{2}(\gamma)}{\partial \theta} & \frac{\partial h_{2}(\gamma)}{\partial \gamma}
\end{array}\right]
$$

Where the pratial derivative are getting as follows:

$$
\begin{aligned}
& \frac{\partial f_{2}(\delta)}{\partial \delta}=\frac{\partial^{2} \sum_{i=1}^{n} \epsilon_{i}^{2}}{\partial^{2} \delta} \\
& \frac{\partial f_{2}(\delta)}{\partial \delta}=-2 \sum_{i=1}^{n}\left[\left\{\frac{n-i+0.5}{n} e^{\theta \delta\left(1-e^{\left(\frac{x_{i}}{\delta}\right)^{\gamma}}\right)}\left(\theta\left(1-e^{\left(\frac{x_{i}}{\delta}\right)^{\gamma}}\right)+\theta \gamma e^{\left(\frac{x_{i}}{\delta}\right)^{\gamma}}\left(\frac{x_{i}}{\delta}\right)^{\gamma}\right)\right.\right. \\
& \left.-e^{2 \theta \delta\left(1-e^{\left(\frac{x_{i}}{\delta}\right)^{\gamma}}\right)}\left(2 \theta\left(1-e^{\left(\frac{x_{i}}{\delta}\right)^{\gamma}}\right)+2 \theta \gamma e^{\left(\frac{x_{i}}{\delta}\right)^{\gamma}}\left(\frac{x_{i}}{\delta}\right)^{\gamma}\right)\right\}\left\{\theta\left(1-e^{\left(\frac{x_{i}}{\delta}\right)^{\gamma}}\right)+\theta \gamma\right. \\
& \left.e^{\left(\frac{x_{i}}{\delta}\right)^{\gamma}}\left(\frac{x_{i}}{\delta}\right)^{\gamma}\right\}+\left\{\frac{n-i+0.5}{n} e^{\theta \delta\left(1-e^{\left(\frac{x_{i}}{\delta}\right)^{\gamma}}\right)}-e^{2 \theta \delta\left(1-e^{\left(\frac{x_{i}}{\delta}\right)^{\gamma}}\right.}\right\}\left\{\frac{\theta \gamma}{\delta} e^{\left(\frac{x_{i}}{\delta}\right)^{\gamma}}\left(\frac{x_{i}}{\delta}\right)^{\gamma}\right. \\
& \left.\left.-\frac{\theta \gamma^{2}}{\delta} e^{\left(\frac{x_{i}}{\delta}\right)^{\gamma}}\left(\frac{x_{i}}{\delta}\right)^{2 \gamma}-\frac{\theta \gamma^{2}}{\delta} e^{\left(\frac{x_{i}}{\delta}\right)^{\gamma}}\left(\frac{x_{i}}{\delta}\right)^{\gamma}\right\}\right] \\
& \frac{\partial f_{2}(\delta)}{\partial \theta}=\frac{\partial^{2} \sum_{i=1}^{n} \epsilon_{i}^{2}}{\partial \theta \partial \delta} \\
& \frac{\partial f_{2}(\delta)}{\partial \theta}=-2 \sum_{i=1}^{n}\left[-e^{2 \theta \delta\left(1-e^{\left(\frac{x_{i}}{\delta}\right)^{\gamma}}\right)} \delta\left(1-e^{\left(\frac{x_{i}}{\delta}\right)^{\gamma}}\right)\left(\theta\left(1-e^{\left(\frac{x_{i}}{\delta}\right)^{\gamma}}\right)+\theta \gamma e^{\left(\frac{x_{i}}{\delta}\right)^{\gamma}}\right.\right. \\
& \left.\left(\frac{x_{i}}{\delta}\right)^{\gamma}\right)+\left(\frac{n-i+0.5}{n}-e^{\theta \delta\left(1-e^{\left(\frac{x_{i}}{\delta}\right)^{\gamma}}\right)}\right) e^{\theta \delta\left(1-e^{\left(\frac{x_{i}}{\delta}\right)^{\gamma}}\right)} \delta\left(1-e^{\left(\frac{x_{i}}{\delta}\right)^{\gamma}}\right)\left(\theta\left(1-e^{\left(\frac{x_{i}}{\delta}\right)^{\gamma}}\right)\right. \\
& \left.+\theta \gamma e^{\left(\frac{x_{i}}{\delta}\right)^{\gamma}}\left(\frac{x_{i}}{\delta}\right)^{\gamma}\right)+\left(\frac{n-i+0.5}{n}-e^{\theta \delta\left(1-e^{\left(\frac{x_{i}}{\delta}\right)^{\gamma}}\right)}\right) e^{\theta \delta\left(1-e^{\left(\frac{x_{i}}{\delta}\right)^{\gamma}}\right)}\left(1-e^{\left(\frac{x_{i}}{\delta}\right)^{\gamma}}\right)+ \\
& \left.+\left(\frac{n-i+0.5}{n}-e^{\theta \delta\left(1-e^{\left(\frac{x_{i}}{\delta}\right)^{\gamma}}\right)}\right) e^{\theta \delta\left(1-e^{\left(\frac{x_{i}}{\delta}\right)^{\gamma}}\right)} \gamma e^{\left(\frac{x_{i}}{\delta}\right)^{\gamma}}\left(\frac{x_{i}}{\delta}\right)^{\gamma}\right] \\
& \frac{\partial f_{2}(\delta)}{\partial \gamma}=\frac{\partial^{2} \sum_{i=1}^{n} \epsilon_{i}^{2}}{\partial \gamma \partial \delta}
\end{aligned}
$$




$$
\begin{aligned}
& \frac{\partial f_{2}(\delta)}{\partial \gamma}=2 \theta \sum_{i=1}^{n}\left[\frac{n-i+0.5}{n} e^{\theta \delta\left(1-e^{\left(\frac{x_{i}}{\delta}\right)^{\gamma}}\right)} \theta \delta e^{\left(\frac{x_{i}}{\delta}\right)^{\gamma}}\left(\frac{x_{i}}{\delta}\right)^{\gamma} \operatorname{Ln}\left(\frac{x_{i}}{\delta}\right)(1\right. \\
& \left.-e^{\left(\frac{x_{i}}{\delta}\right)^{\gamma}}\right)+\frac{n-i+0.5}{n} e^{\theta \delta\left(1-e^{\left(\frac{x_{i}}{\delta}\right)^{\gamma}}\right)} \theta \delta \gamma e^{2\left(\frac{x_{i}}{\delta}\right)^{\gamma}}\left(\frac{x_{i}}{\delta}\right)^{2 \gamma} \operatorname{Ln}\left(\frac{x_{i}}{\delta}\right)-2 \\
& e^{2 \theta \delta\left(1-e^{\left(\frac{x_{i}}{\delta}\right)^{\gamma}}\right)} \theta \delta e^{\left(\frac{x_{i}}{\delta}\right)^{\gamma}}\left(\frac{x_{i}}{\delta}\right)^{\gamma} \operatorname{Ln}\left(\frac{x_{i}}{\delta}\right)\left(1-e^{\left(\frac{x_{i}}{\delta}\right)^{\gamma}}\right)-2 \theta \delta \gamma e^{2 \theta \delta\left(1-e^{\left(\frac{x_{i}}{\delta}\right)^{\gamma}}\right)} \\
& e^{2\left(\frac{x_{i}}{\delta}\right)^{\gamma}}\left(\frac{x_{i}}{\delta}\right)^{2 \gamma} \operatorname{Ln}\left(\frac{x_{i}}{\delta}\right)+\frac{n-i+0.5}{n} e^{\theta \delta\left(1-e^{\left(\frac{x_{i}}{\delta}\right)^{\gamma}}\right)} e^{\left(\frac{x_{i}}{\delta}\right)^{\gamma}}\left(\frac{x_{i}}{\delta}\right)^{\gamma} \operatorname{Ln}\left(\frac{x_{i}}{\delta}\right)-
\end{aligned}
$$

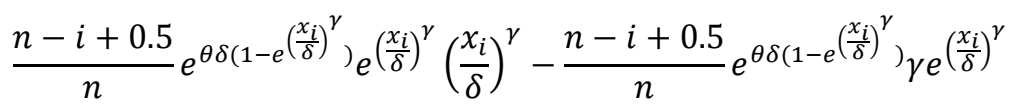

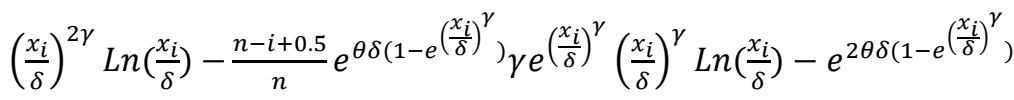

$$
\begin{aligned}
& e^{\left(\frac{x_{i}}{\delta}\right)^{\gamma}}\left(\frac{x_{i}}{\delta}\right)^{\gamma} \operatorname{Ln}\left(\frac{x_{i}}{\delta}\right)+e^{2 \theta \delta\left(1-e^{\left(\frac{x_{i}}{\delta}\right)^{\gamma}}\right)} e^{\left(\frac{x_{i}}{\delta}\right)^{\gamma}}\left(\frac{x_{i}}{\delta}\right)^{\gamma}+\gamma e^{2 \theta \delta\left(1-e^{\left(\frac{x_{i}}{\delta}\right)^{\gamma}}\right)} e^{\left(\frac{x_{i}}{\delta}\right)^{\gamma}} \\
& \left.\left(\frac{x_{i}}{\delta}\right)^{2 \gamma} \operatorname{Ln}\left(\frac{x_{i}}{\delta}\right)+\gamma e^{2 \theta \delta\left(1-e^{\left(\frac{x_{i}}{\delta}\right)^{\gamma}}\right)} e^{\left(\frac{x_{i}}{\delta}\right)^{\gamma}}\left(\frac{x_{i}}{\delta}\right)^{\gamma} \operatorname{Ln}\left(\frac{x_{i}}{\delta}\right)\right] \\
& \frac{\partial g_{2}(\theta)}{\partial \delta}=\frac{\partial^{2} \sum_{i=1}^{n} \epsilon_{i}^{2}}{\partial \delta \partial \theta}=\frac{\partial f_{2}(\delta)}{\partial \theta} \\
& \frac{\partial g_{2}(\theta)}{\partial \theta}=\frac{\partial^{2} \sum_{i=1}^{n} \epsilon_{i}^{2}}{\partial \theta^{2}} \\
& \frac{\partial g_{2}(\theta)}{\partial \theta}=-2 \delta^{2} \sum_{i=1}^{n}\left(1-e^{\left(\frac{x_{i}}{\delta}\right)^{\gamma}}\right)^{2} e^{\theta \delta\left(1-e^{\left(\frac{x_{i}}{\delta}\right)^{\gamma}}\right)}\left[\frac{n-i+0.5}{n}-2 e^{\theta \delta\left(1-e^{\left(\frac{x_{i}}{\delta}\right)^{\gamma}}\right)}\right] \\
& \frac{\partial g_{2}(\theta)}{\partial \gamma}=\frac{\partial^{2} \sum_{i=1}^{n} \epsilon_{i}^{2}}{\partial \gamma \partial \theta} \\
& \frac{\partial g_{2}(\theta)}{\partial \gamma}=2 \delta \sum_{i=1}^{n}\left[\frac{n-i+0.5}{n} \theta \delta e^{\left(\frac{x_{i}}{\delta}\right)^{\gamma}}\left(\frac{x_{i}}{\delta}\right)^{\gamma} \operatorname{Ln}\left(\frac{x_{i}}{\delta}\right)\left(1-e^{\left(\frac{x_{i}}{\delta}\right)^{\gamma}}\right)\right. \\
& e^{\theta \delta\left(1-e^{\left(\frac{x_{i}}{\delta}\right)^{\gamma}}\right)}-2 \theta \delta e^{2 \theta \delta\left(1-e^{\left(\frac{x_{i}}{\delta}\right)^{\gamma}}\right)} e^{\left(\frac{x_{i}}{\delta}\right)^{\gamma}}\left(\frac{x_{i}}{\delta}\right)^{\gamma} \operatorname{Ln}\left(\frac{x_{i}}{\delta}\right)\left(1-e^{\left(\frac{x_{i}}{\delta}\right)^{\gamma}}\right)+ \\
& \left.\frac{n-i+0.5}{n} e^{\theta \delta\left(1-e^{\left(\frac{x_{i}}{\delta}\right)^{\gamma}}\right)} e^{\left(\frac{x_{i}}{\delta}\right)^{\gamma}}\left(\frac{x_{i}}{\delta}\right)^{\gamma} \operatorname{Ln}\left(\frac{x_{i}}{\delta}\right)-e^{2 \theta \delta\left(1-e^{\left(\frac{x_{i}}{\delta}\right)^{\gamma}}\right)} e^{\left(\frac{x_{i}}{\delta}\right)^{\gamma}}\left(\frac{x_{i}}{\delta}\right)^{\gamma} \operatorname{Ln}\left(\frac{x_{i}}{\delta}\right)\right]
\end{aligned}
$$




$$
\begin{aligned}
& \frac{\partial h_{2}(\gamma)}{\partial \delta}=\frac{\partial^{2} \sum_{i=1}^{n} \epsilon_{i}^{2}}{\partial \delta \partial \gamma}=\frac{\partial f_{2}(\delta)}{\partial \gamma} \\
& \frac{\partial h_{2}(\gamma)}{\partial \theta}=\frac{\partial^{2} \sum_{i=1}^{n} \epsilon_{i}^{2}}{\partial \theta \partial \gamma}=\frac{\partial g_{2}(\theta)}{\partial \gamma} \\
& \frac{\partial h_{2}(\gamma)}{\partial \gamma}=\frac{\partial^{2} \sum_{i=1}^{n} \epsilon_{i}^{2}}{\partial \gamma^{2}} \\
& \frac{\partial h_{2}(\gamma)}{\partial \gamma}=2 \theta \delta \sum_{i=1}^{n} \operatorname{Ln}\left(\frac{x_{i}}{\delta}\right)\left[\left\{-\theta \delta\left(\frac{n-i+0.5}{n}\right) e^{\theta \delta\left(1-e^{\left(\frac{x_{i}}{\delta}\right)^{\gamma}}\right)} e^{2\left(\frac{x_{i}}{\delta}\right)^{\gamma}}\left(\frac{x_{i}}{\delta}\right)^{\gamma} \operatorname{Ln}\left(\frac{x_{i}}{\delta}\right)\right.\right. \\
& +\frac{n-i+0.5}{n} e^{\theta \delta\left(1-e^{\left(\frac{x_{i}}{\delta}\right)^{\gamma}}\right)} e^{\left(\frac{x_{i}}{\delta}\right)^{\gamma}}\left(\frac{x_{i}}{\delta}\right)^{\gamma} \operatorname{Ln}\left(\frac{x_{i}}{\delta}\right)+2 \theta \delta e^{2 \theta \delta\left(1-e^{\left(\frac{x_{i}}{\delta}\right)^{\gamma}}\right)} \\
& \left.e^{2\left(\frac{x_{i}}{\delta}\right)^{\gamma}}\left(\frac{x_{i}}{\delta}\right)^{\gamma} \operatorname{Ln}\left(\frac{x_{i}}{\delta}\right)-e^{2 \theta \delta\left(1-e^{\left(\frac{x_{i}}{\delta}\right)^{\gamma}}\right)} e^{\left(\frac{x_{i}}{\delta}\right)^{\gamma}}\left(\frac{x_{i}}{\delta}\right)^{\gamma} \operatorname{Ln}\left(\frac{x_{i}}{\delta}\right)\right\}\left\{\left(\frac{x_{i}}{\delta}\right)^{\gamma}\right\} \\
& +\frac{n-i+0.5}{n} e^{\theta \delta\left(1-e^{\left(\frac{x_{i}}{\delta}\right)^{\gamma}}\right)} e^{\left(\frac{x_{i}}{\delta}\right)^{\gamma}}\left(\frac{x_{i}}{\delta}\right)^{\gamma} \operatorname{Ln}\left(\frac{x_{i}}{\delta}\right)-e^{2 \theta \delta\left(1-e^{\left(\frac{x_{i}}{\delta}\right)^{\gamma}}\right)} e^{\left(\frac{x_{i}}{\delta}\right)^{\gamma}} \\
& \left.\left(\frac{x_{i}}{\delta}\right)^{\gamma} \operatorname{Ln}\left(\frac{x_{i}}{\delta}\right)\right]
\end{aligned}
$$

Thus, all above equations in section 4 , proved that the Jacobian matrix $J_{K 2}$ is non-singular symmetric matrix, therefore:

$$
\left[\begin{array}{l}
\delta_{k+1} \\
\theta_{k+1} \\
\gamma_{k+1}
\end{array}\right]=\left[\begin{array}{l}
\delta_{k} \\
\theta_{k} \\
\gamma_{k}
\end{array}\right]-J_{K 2}{ }^{-1}\left[\begin{array}{l}
f_{2}\left(\delta_{k}\right) \\
g_{2}\left(\theta_{k}\right) \\
h_{2}\left(\gamma_{k}\right)
\end{array}\right]
$$

\section{Simulation Results}

The Monte Carlo method is most popular method in simulation techniques using to generate the observations (samples) for any distribution. The simulation process is flexibility, and gave ability for the test and experimenting by frequency many time.

1- To apply Monte Carlo method for modified Weibull extension, we use the cumulative distribution function as follows:

$$
F(x)=1-e^{\theta \delta\left(1-e^{\left(\frac{x}{\delta}\right)^{\gamma}}\right)}
$$

By substitute $F(x)$ as $u$, with a random number, then $u=F(x)$, that is $x=F^{-1}(u)$. Thus

$$
\begin{gathered}
e^{\theta \delta\left(1-e^{\left(\frac{x}{\delta}\right)^{\gamma}}\right)}=1-u \\
\theta \delta\left(1-e^{\left(\frac{x}{\delta}\right)^{\gamma}}\right)=\operatorname{Ln}(1-u)
\end{gathered}
$$




$$
\begin{gathered}
\left(1-e^{\left(\frac{x}{\delta}\right)^{\gamma}}\right)=\frac{\operatorname{Ln}(1-u)}{\theta \delta} \\
e^{\left(\frac{x}{\delta}\right)^{\gamma}}=1-\frac{\operatorname{Ln}(1-u)}{\theta \delta} \\
\left(\frac{x}{\delta}\right)^{\gamma}=\operatorname{Ln}\left(1-\frac{\operatorname{Ln}(1-u)}{\theta \delta}\right) \\
x^{\gamma}=\delta^{\gamma} \operatorname{Ln}\left(1-\frac{\operatorname{Ln}(1-u)}{\theta \delta}\right) \\
x=\left(\delta^{\gamma} \operatorname{Ln}\left(1-\frac{\operatorname{Ln}(1-u)}{\theta \delta}\right)\right)^{\frac{1}{\gamma}}
\end{gathered}
$$

2- Selected many different sample size

$(\mathrm{n}=10,20,30,50,100)$.

3- Replicate to each experiment as ( $\mathrm{m}=1000,2500,5000)$.

4- Plot a system of six-nonlinear equations,

$\left[\begin{array}{l}f_{1}(\delta) \\ g_{1}(\theta) \\ h_{1}(\gamma) \\ f_{2}(\delta) \\ g_{2}(\theta) \\ h_{2}(\gamma)\end{array}\right]=\left[\begin{array}{l}0 \\ 0 \\ 0 \\ 0 \\ 0 \\ 0\end{array}\right]$, For one of above sample sizes, for example, taking samples sizes $(\mathrm{n}=100)$, and sample replications $(\mathrm{m}=5000)$, can assist in finding initial approximations for both estimator methods, to find the values of parameters in two estimator methods. The plot is depicted in Fig.1.

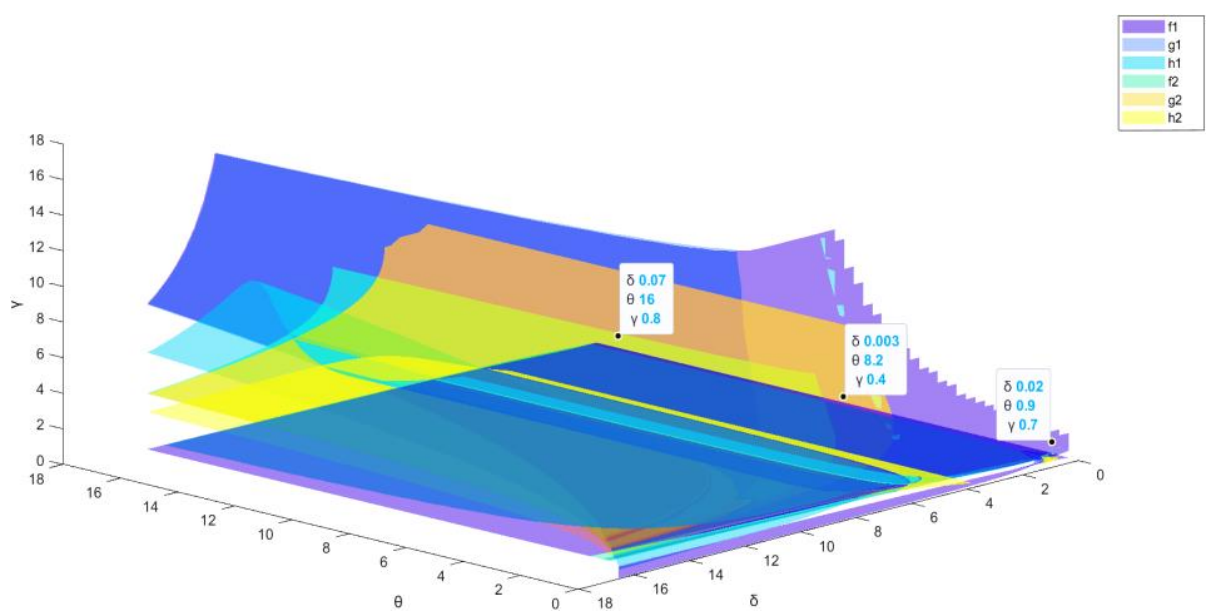

Fig. 1. Initial approximations for both estimator methods.

5 -Selected the assumed values for the parameters

$\boldsymbol{\delta}=0.07,0.003,0.02$

$\boldsymbol{\theta}=16.0,8.2,0.9$

$\gamma=0.8,0.4,0.7$.

6- To employ mesure of mean square error to compare between two methods of estimation given by the following formula $\operatorname{MSE}(\hat{\theta})=\frac{1}{m} \sum_{i=1}^{m}\left(\widehat{\theta}_{l}-\theta\right)^{2}$, where $\hat{\theta}$ is the estimate of $\delta, \theta, \gamma$ 
7- For illustrating the basics of the process in MLE, explaining the first iteration for finding the first estimating of $\boldsymbol{\delta}_{\mathbf{1}}, \boldsymbol{\theta}_{\mathbf{1}}, \boldsymbol{\gamma}_{\mathbf{1}}$, at first initial guess in Simulink as follows, with $\mathrm{m}=5000$, $\mathrm{n}=100$

Finding $J_{K 1}$ at first initial guess $\boldsymbol{\delta}_{\mathbf{0}}=\mathbf{0 . 0 2}, \boldsymbol{\theta}_{\mathbf{0}}=\mathbf{0 . 9}, \boldsymbol{\gamma}_{\mathbf{0}}=\mathbf{0 . 7}$, And finding $\left[\begin{array}{l}f_{1}(\delta) \\ g_{1}(\theta) \\ h_{1}(\gamma)\end{array}\right]$ at

first initial guess $\boldsymbol{\delta}_{\mathbf{0}}, \boldsymbol{\theta}_{\mathbf{0}}, \boldsymbol{\gamma}_{\mathbf{0}}$, And applying

$J_{K 1}\left[\begin{array}{l}\boldsymbol{\delta}_{\mathbf{1}} \\ \boldsymbol{\theta}_{\mathbf{1}} \\ \gamma_{\mathbf{1}}\end{array}\right]-\left[\begin{array}{l}f_{1}(\delta) \\ g_{1}(\theta) \\ h_{1}(\gamma)\end{array}\right]=0$, which determined as $f(z)=\left[\begin{array}{c}a_{1} \boldsymbol{\delta}_{\mathbf{1}}+a_{2} \boldsymbol{\delta}_{\mathbf{1}} a_{3} \boldsymbol{\delta}_{\mathbf{1}} a_{4} \\ a_{5} \boldsymbol{\theta}_{\mathbf{1}}+a_{6} \boldsymbol{\theta}_{\mathbf{1}}+a_{7} \boldsymbol{\theta}_{\mathbf{1}}+a_{8} \\ a_{9} \boldsymbol{\gamma}_{\mathbf{1}}+a_{10} \boldsymbol{\gamma}_{\mathbf{1}}+a_{11} \boldsymbol{\gamma}_{\mathbf{1}}+a_{12}\end{array}\right]=0$

After solving the above equation, adding the result with the first initial guess, to obtain the estimator for the first parameter $\boldsymbol{\delta}_{\mathbf{1}}$, the second parameter $\boldsymbol{\theta}_{\mathbf{1}}$, and the third parameter $\boldsymbol{\gamma}_{\mathbf{1}}$, then finding the first iteration of mean square error for parameters, the process is depicted in Fig.2. By the same way for the process in OLS.

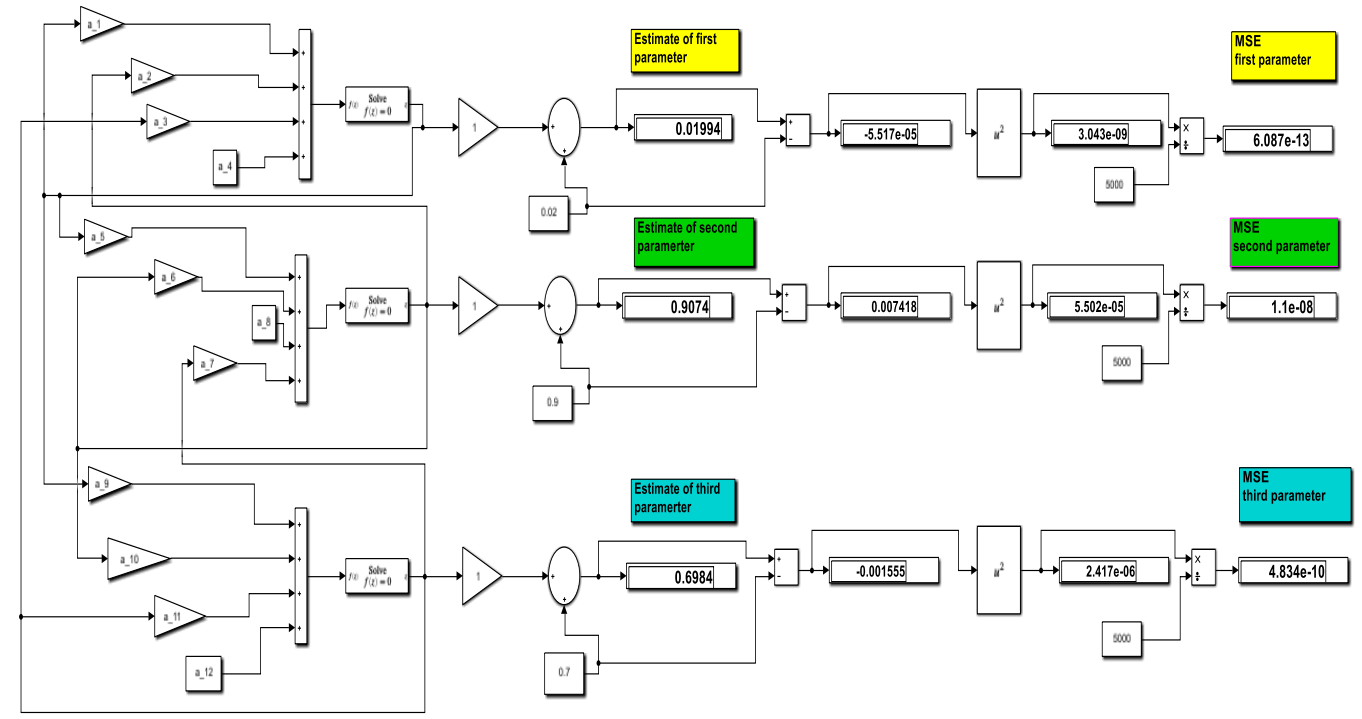

Fig. 2. The process in MLE, for the first iteration. 
Table 1. MSE for the estimation of $\boldsymbol{\delta}$ at $\mathbf{E} 1\left\{{ }^{\prime} \boldsymbol{\delta}=\mathbf{0 . 0 2}\right\} \quad\left\{{ }^{\prime} \boldsymbol{\theta}=\mathbf{0 . 9}^{\prime}\right\} \quad\left\{{ }^{\prime} \boldsymbol{\gamma}=\mathbf{0 . 7}\right\}$.

\begin{tabular}{|c|c|c|c|c|}
\hline $\mathrm{m}$ & $\mathrm{n}$ & MLE & OLS & best \\
\hline \multirow{4}{*}{1000} & 10 & 7.4699 e-09 & $1.5353 \mathrm{e}-05$ & MLE \\
\cline { 2 - 5 } & 20 & 7.7755 e-09 & 1.5102 e-05 & MLE \\
\cline { 2 - 5 } & 30 & 7.8831 e-09 & 1.4979 e-05 & MLE \\
\cline { 2 - 5 } & $\mathbf{5 0}$ & 7.6583 e-09 & 1.4895 e-05 & MLE \\
\cline { 2 - 5 } & 100 & 7.6147 e-09 & 1.4833 e-05 & MLE \\
\hline \multirow{4}{*}{2500} & 10 & 7.5737 e-09 & 1.5424 e-05 & MLE \\
\cline { 2 - 5 } & 20 & 7.7216 e-09 & 1.5101 e-05 & MLE \\
\cline { 2 - 5 } & 30 & 7.593 e-09 & 1.4972 e-05 & MLE \\
\cline { 2 - 5 } & $\mathbf{5 0}$ & 7.579 e-09 & 1.4853 e-05 & MLE \\
\cline { 2 - 5 } & 100 & 7.7307 e-09 & 1.4796 e-05 & MLE \\
\hline \multirow{4}{*}{5000} & 10 & 7.7353 e-09 & 1.5498 e-05 & MLE \\
\cline { 2 - 5 } & 20 & 7.6006 e-09 & 1.5054 e-05 & MLE \\
\cline { 2 - 5 } & 30 & 7.5871 e-09 & 1.4974 e-05 & MLE \\
\cline { 2 - 5 } & 50 & 7.7125 e-09 & 1.4878 e-05 & MLE \\
\cline { 2 - 5 } & 100 & 7.6811 e-09 & 1.4812 e-05 & MLE \\
\hline
\end{tabular}

Table 2. MSE for the estimation of $\boldsymbol{\theta}$ at $\mathbf{E} 1\left\{{ }^{\prime} \boldsymbol{\delta}=\mathbf{0 . 0 2}\right\} \quad\left\{{ }^{\prime} \boldsymbol{\theta}=\mathbf{0 . 9}^{\prime}\right\} \quad\left\{{ }^{\prime} \boldsymbol{\gamma}=\mathbf{0 . 7}\right\}$.

\begin{tabular}{|c|c|c|c|c|}
\hline $\mathbf{m}$ & $\mathbf{n}$ & MLE & OLS & best \\
\hline \multirow{4}{*}{1000} & $\mathbf{1 0}$ & $\mathbf{0 . 0 0 0 1 0 0 5 1}$ & $\mathbf{0 . 1 9 6 2 3}$ & MLE \\
\cline { 2 - 5 } & $\mathbf{2 0}$ & $\mathbf{0 . 0 0 0 1 0 3 1 3}$ & $\mathbf{0 . 1 9 6 9}$ & MLE \\
\cline { 2 - 5 } & $\mathbf{3 0}$ & $\mathbf{0 . 0 0 0 1 0 3 9 6}$ & $\mathbf{0 . 1 9 7 3 7}$ & MLE \\
\cline { 2 - 5 } & $\mathbf{5 0}$ & $\mathbf{0 . 0 0 0 1 0 2 0 1}$ & $\mathbf{0 . 1 9 7 5 6}$ & MLE \\
\cline { 2 - 5 } & $\mathbf{1 0 0}$ & $\mathbf{0 . 0 0 0 1 0 1 8 9}$ & $\mathbf{0 . 1 9 7 7 3}$ & MLE \\
\hline \multirow{4}{*}{2500} & $\mathbf{1 0}$ & $\mathbf{0 . 0 0 0 1 0 1 5 5}$ & $\mathbf{0 . 1 9 5 8 8}$ & MLE \\
\cline { 2 - 5 } & $\mathbf{2 0}$ & $\mathbf{0 . 0 0 0 1 0 2 7 3}$ & $\mathbf{0 . 1 9 6 9 2}$ & MLE \\
\cline { 2 - 5 } & $\mathbf{3 0}$ & $\mathbf{0 . 0 0 0 1 0 1 7 5}$ & $\mathbf{0 . 1 9 7 2 4}$ & MLE \\
\cline { 2 - 5 } & $\mathbf{5 0}$ & $\mathbf{0 . 0 0 0 1 0 1 6 3}$ & $\mathbf{0 . 1 9 7 6 1}$ & MLE \\
\cline { 2 - 5 } & $\mathbf{1 0 0}$ & $\mathbf{0 . 0 0 0 1 0 3 0 1}$ & $\mathbf{0 . 1 9 7 8 8}$ & MLE \\
\hline \multirow{5}{*}{5000} & $\mathbf{1 0}$ & $\mathbf{0 . 0 0 0 1 0 2 7 6}$ & $\mathbf{0 . 1 9 5 8}$ & MLE \\
\cline { 2 - 5 } & $\mathbf{2 0}$ & $\mathbf{0 . 0 0 0 1 0 1 6 4}$ & $\mathbf{0 . 1 9 7 0 9}$ & MLE \\
\cline { 2 - 5 } & $\mathbf{3 0}$ & $\mathbf{0 . 0 0 0 1 0 1 6 9}$ & $\mathbf{0 . 1 9 7 3}$ & MLE \\
\cline { 2 - 5 } & $\mathbf{5 0}$ & $\mathbf{0 . 0 0 0 1 0 2 6 4}$ & $\mathbf{0 . 1 9 6 3}$ & MLE \\
\cline { 2 - 5 } & $\mathbf{1 0 0}$ & $\mathbf{0 . 0 0 0 1 0 2 4 2}$ & $\mathbf{0 . 1 9 7 8 3}$ & MLE \\
\hline
\end{tabular}


Table 3. MSE for the estimation of $\boldsymbol{\gamma}$ at $\mathbf{E} 1\left\{{ }^{\prime} \boldsymbol{\delta}=\mathbf{0 . 0 2}\right\} \quad\left\{{ }^{\prime} \boldsymbol{\theta}=\mathbf{0 . 9}^{\prime}\right\} \quad\left\{{ }^{\prime} \boldsymbol{\gamma}=\mathbf{0 . 7}\right\}$.

\begin{tabular}{|c|c|c|c|c|}
\hline $\mathbf{m}$ & $\mathbf{n}$ & MLE & OLS & best \\
\hline \multirow{5}{*}{1000} & 10 & $5.5494 \mathrm{e}-06$ & 0.011528 & MLE \\
\hline & 20 & $5.7568 \mathrm{e}-06$ & 0.011417 & MLE \\
\hline & 30 & $5.8284 \mathrm{e}-06$ & 0.011367 & MLE \\
\hline & 50 & $5.6752 \mathrm{e}-06$ & 0.011326 & MLE \\
\hline & 100 & 5.6499 e- 06 & 0.011296 & MLE \\
\hline \multirow{5}{*}{2500} & 10 & $5.6224 \mathrm{e}-06$ & 0.011555 & MLE \\
\hline & 20 & $5.7213 \mathrm{e}-06$ & 0.011418 & MLE \\
\hline & 30 & $5.6361 \mathrm{e}-06$ & 0.011356 & MLE \\
\hline & 50 & $5.6267 \mathrm{e}-06$ & 0.011302 & MLE \\
\hline & 100 & $5.7311 \mathrm{e}-06$ & 0.011278 & MLE \\
\hline \multirow{5}{*}{5000} & 10 & $5.7288 \mathrm{e}-06$ & 0.011596 & MLE \\
\hline & 20 & $5.6381 \mathrm{e}-06$ & 0.011395 & MLE \\
\hline & 30 & $5.6318 \mathrm{e}-06$ & 0.011359 & MLE \\
\hline & 50 & 5.715 e-06 & 0.011317 & MLE \\
\hline & 100 & $5.6945 \mathrm{e}-06$ & 0.011287 & MLE \\
\hline
\end{tabular}

Table 4. MSE for the estimation of $\delta$ at $\mathbf{E 2} \quad\left\{{ }^{\prime} \boldsymbol{\delta}=\mathbf{0 . 0 0 3}\right\} \quad\left\{{ }^{\prime} \boldsymbol{\theta}=\mathbf{8 . 2}\right\} \quad\left\{{ }^{\prime} \boldsymbol{\gamma}=\mathbf{0 . 4} \mathbf{4}^{\prime}\right\}$

\begin{tabular}{|c|c|c|c|c|}
\hline m & n & MLE & OLS & best \\
\hline \multirow{4}{*}{1000} & 10 & 4.151 e-09 & 9.7399 e-07 & MLE \\
\cline { 2 - 5 } & 20 & 4.265 e-09 & 9.6521 e-07 & MLE \\
\cline { 2 - 5 } & 30 & 4.2976 e-09 & 9.6219 e-07 & MLE \\
\cline { 2 - 5 } & 50 & 4.2103 e-09 & 9.5829 e-07 & MLE \\
\cline { 2 - 5 } & 100 & 4.2078 e-09 & 9.5573 e-07 & MLE \\
\hline \multirow{5}{*}{2500} & 10 & 4.1923 e-09 & 9.7461 e-07 & MLE \\
\cline { 2 - 5 } & 20 & 4.2451 e-09 & 9.6549 e-07 & MLE \\
\cline { 2 - 5 } & 30 & 4.2011 e-09 & 9.598 e-07 & MLE \\
\cline { 2 - 5 } & 50 & 4.1966 e-09 & 9.558 e-07 & MLE \\
\cline { 2 - 5 } & 100 & 4.2524 e-09 & 9.5453 e-07 & MLE \\
\hline \multirow{5}{*}{5000} & 10 & 4.2445 e-09 & 9.7905 e-07 & MLE \\
\cline { 2 - 5 } & 20 & 4.1962 e-09 & 9.637 e-07 & MLE \\
\cline { 2 - 5 } & 30 & 4.1972 e-09 & 9.6058 e-07 & MLE \\
\cline { 2 - 5 } & $\mathbf{5 0}$ & 4.2403 e-09 & 9.5771 e-07 & MLE \\
\cline { 2 - 5 } & 100 & 4.2296 e-09 & 9.5536 e-07 & MLE \\
\hline
\end{tabular}




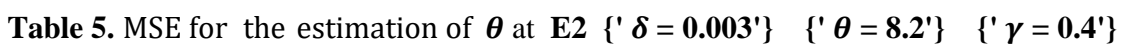

\begin{tabular}{|c|c|c|c|c|}
\hline $\mathbf{m}$ & $\mathbf{n}$ & MLE & OLS & best \\
\hline \multirow{5}{*}{1000} & 10 & 0.084508 & 1.675 & MLE \\
\hline & 20 & 0.08583 & 1.723 & MLE \\
\hline & 30 & 0.086261 & 1.747 & MLE \\
\hline & 50 & $\mathbf{0 . 0 8 5 1 3 8}$ & 1.768 & MLE \\
\hline & 100 & $\mathbf{0 . 0 8 5 3 5}$ & 1.78 & MLE \\
\hline \multirow{5}{*}{2500} & 10 & 0.085168 & 1.671 & MLE \\
\hline & 20 & $\mathbf{0 . 0 8 5 7 9 7}$ & 1.722 & MLE \\
\hline & 30 & $\mathbf{0 . 0 8 5 2 9 1}$ & 1.753 & MLE \\
\hline & 50 & 0.085382 & 1.774 & MLE \\
\hline & 100 & 0.085841 & 1.79 & MLE \\
\hline \multirow{5}{*}{5000} & 10 & 0.08567 & 1.655 & MLE \\
\hline & 20 & $\mathbf{0 . 0 8 5 2 1 5}$ & 1.735 & MLE \\
\hline & 30 & 0.08517 & 1.751 & MLE \\
\hline & 50 & 0.085642 & 1.77 & MLE \\
\hline & 100 & 0.085582 & 1.783 & MLE \\
\hline
\end{tabular}

Table 6. MSE for the estimation of $\boldsymbol{\gamma}$ at $\mathbf{E 2} \quad\left\{{ }^{\prime} \boldsymbol{\delta}=\mathbf{0 . 0 0 3}^{\prime}\right\} \quad\left\{{ }^{\prime} \boldsymbol{\theta}=8.2^{\prime}\right\} \quad\left\{{ }^{\prime} \boldsymbol{\gamma}=\mathbf{0 . 4} \mathbf{4}^{\prime}\right\}$

\begin{tabular}{|c|c|c|c|c|}
\hline $\mathbf{m}$ & $\mathbf{n}$ & MLE & OLS & best \\
\hline \multirow{5}{*}{1000} & 10 & $3.0392 \mathrm{e}-05$ & 0.0063268 & MLE \\
\hline & 20 & $3.1084 \mathrm{e}-05$ & 0.0063016 & MLE \\
\hline & 30 & 3.1287 e- 05 & 0.0062922 & MLE \\
\hline & 50 & 3.0746 e-05 & 0.0062786 & MLE \\
\hline & 100 & 3.0759 e-05 & 0.0062706 & MLE \\
\hline \multirow{5}{*}{2500} & 10 & 3.0666 e- 05 & 0.0063294 & MLE \\
\hline & 20 & $3.0983 \mathrm{e}-05$ & 0.006303 & MLE \\
\hline & 30 & 3.0723 e- 05 & 0.0062827 & MLE \\
\hline & 50 & 3.0705 e- 05 & 0.0062693 & MLE \\
\hline & 100 & 3.1034 e- 05 & 0.0062654 & MLE \\
\hline \multirow{5}{*}{5000} & 10 & 3.0968 e- 05 & 0.006346 & MLE \\
\hline & 20 & 3.0684 e-05 & 0.0062955 & MLE \\
\hline & 30 & 3.0694 e- 05 & 0.006286 & MLE \\
\hline & 50 & 3.0947 e- 05 & 0.0062767 & MLE \\
\hline & 100 & $3.0888 \mathrm{e}-05$ & 0.0062691 & MLE \\
\hline
\end{tabular}


Table 7. MSE for the estimation of $\boldsymbol{\delta}$ at E3 $\quad\left\{\boldsymbol{\delta}^{\mathrm{s}}=0.07^{\prime}\right\} \quad\left\{{ }^{\prime} \boldsymbol{\theta}=16.0^{\prime}\right\} \quad\left\{{ }^{\prime} \boldsymbol{\gamma}=0.8^{\prime}\right\}$.

\begin{tabular}{|c|c|c|c|c|}
\hline $\mathbf{m}$ & $\mathbf{n}$ & MLE & OLS & best \\
\hline \multirow{5}{*}{1000} & 10 & 3.0821 e-07 & 0.00034378 & MLE \\
\hline & 20 & 2.2765 e-07 & 0.000332 & MLE \\
\hline & 30 & 1.9724 e-07 & 0.00032659 & MLE \\
\hline & 50 & 1.7103 e-07 & 0.00032311 & MLE \\
\hline & 100 & 1.468 e-07 & 0.00032067 & MLE \\
\hline \multirow{5}{*}{2500} & 10 & 3.1315 e-07 & 0.00034643 & MLE \\
\hline & 20 & 2.2851 e- 07 & 0.0003319 & MLE \\
\hline & 30 & $1.988 \mathrm{e}-07$ & 0.00032654 & MLE \\
\hline & 50 & 1.7004 e-07 & 0.00032179 & MLE \\
\hline & 100 & 1.4464 e-07 & 0.00031935 & MLE \\
\hline \multirow{5}{*}{5000} & 10 & 3.2276 e-07 & 0.00034953 & MLE \\
\hline & 20 & 2.2595 e-07 & 0.00033012 & MLE \\
\hline & 30 & 1.9861 e-07 & 0.0003266 & MLE \\
\hline & 50 & 1.7072 e-07 & 0.00032264 & MLE \\
\hline & 100 & 1.452 e-07 & 0.00031988 & MLE \\
\hline
\end{tabular}

Table 8. MSE for the estimation of $\boldsymbol{\theta}$ at E3 $\left\{\boldsymbol{~}^{\boldsymbol{\delta}}=0.07^{\prime}\right\} \quad\left\{{ }^{\prime} \boldsymbol{\theta}=16.0^{\prime}\right\} \quad\left\{{ }^{\prime} \boldsymbol{\gamma}=0.8^{\prime}\right\}$.

\begin{tabular}{|c|c|c|c|c|}
\hline m & n & MLE & OLS & best \\
\hline \multirow{4}{*}{1000} & 10 & $\mathbf{0 . 0 6 2 1 6 6}$ & 1.756 & MLE \\
\cline { 2 - 5 } & $\mathbf{2 0}$ & $\mathbf{0 . 0 5 2 5 8 6}$ & $\mathbf{2 . 1 1 3}$ & MLE \\
\cline { 2 - 5 } & $\mathbf{3 0}$ & $\mathbf{0 . 0 4 8 3 6 9}$ & $\mathbf{2 . 3 4 6}$ & MLE \\
\cline { 2 - 5 } & $\mathbf{5 0}$ & $\mathbf{0 . 0 4 4 5 8 8}$ & $\mathbf{2 . 3 9 9}$ & MLE \\
\cline { 2 - 5 } & $\mathbf{1 0 0}$ & $\mathbf{0 . 0 4 0 6 4 6}$ & $\mathbf{2 . 4 8 4}$ & MLE \\
\hline \multirow{4}{*}{2500} & $\mathbf{1 0}$ & $\mathbf{0 . 0 6 2 8 3 4}$ & $\mathbf{1 . 5 7 1}$ & MLE \\
\cline { 2 - 5 } & $\mathbf{2 0}$ & $\mathbf{0 . 0 5 2 7 9}$ & $\mathbf{2 . 0 9 8}$ & MLE \\
\cline { 2 - 5 } & $\mathbf{3 0}$ & $\mathbf{0 . 0 4 8 7 3 1}$ & $\mathbf{2 . 2 3 6}$ & MLE \\
\cline { 2 - 5 } & $\mathbf{5 0}$ & $\mathbf{0 . 0 4 4 3 5 1}$ & $\mathbf{2 . 4 0 2}$ & MLE \\
\cline { 2 - 5 } & $\mathbf{1 0 0}$ & $\mathbf{0 . 0 4 0 1 7 2}$ & $\mathbf{2 . 5 7 2}$ & MLE \\
\hline \multirow{5}{*}{$\mathbf{5 0 0 0}$} & $\mathbf{1 0}$ & $\mathbf{0 . 0 6 3 6 7 5}$ & $\mathbf{1 . 5 6 1}$ & MLE \\
\cline { 2 - 5 } & $\mathbf{2 0}$ & $\mathbf{0 . 0 5 2 4 1 7}$ & $\mathbf{2 . 2 1 4}$ & MLE \\
\cline { 2 - 5 } & $\mathbf{3 0}$ & $\mathbf{0 . 0 4 8 6 9 6}$ & $\mathbf{2 . 2 8 2}$ & MLE \\
\cline { 2 - 5 } & $\mathbf{5 0}$ & $\mathbf{0 . 0 4 4 4 7 2}$ & $\mathbf{2 . 4 5 8}$ & MLE \\
\cline { 2 - 5 } & $\mathbf{1 0 0}$ & $\mathbf{0 . 0 4 0 2 7 9}$ & $\mathbf{2 . 5 4 2}$ & MLE \\
\hline
\end{tabular}




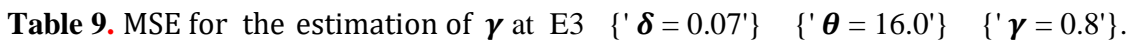

\begin{tabular}{|c|c|c|c|c|}
\hline $\mathbf{m}$ & $\mathbf{n}$ & MLE & OLS & best \\
\hline \multirow{5}{*}{1000} & 10 & 2.1727 e- 05 & 0.024765 & MLE \\
\hline & 20 & 1.6545 e-05 & 0.024225 & MLE \\
\hline & 30 & 1.4526 e- 05 & 0.023989 & MLE \\
\hline & 50 & $1.2769 \mathrm{e}-05$ & 0.023815 & MLE \\
\hline & 100 & 1.1106 e-05 & 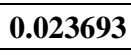 & MLE \\
\hline \multirow{5}{*}{2500} & 10 & 2.2051 e- 05 & 0.024879 & MLE \\
\hline & 20 & 1.6609 e- 05 & 0.024225 & MLE \\
\hline & 30 & $1.4642 \mathrm{e}-05$ & 0.023963 & MLE \\
\hline & 50 & $1.2695 \mathrm{e}-05$ & $\mathbf{0 . 0 2 3 7 3 7}$ & MLE \\
\hline & 100 & 1.0948 e-05 & 0.023629 & MLE \\
\hline \multirow{5}{*}{5000} & 10 & 2.2629 e- 05 & 0.025043 & MLE \\
\hline & 20 & $1.6438 \mathrm{e}-05$ & 0.024136 & MLE \\
\hline & 30 & 1.4629 e-05 & 0.023971 & MLE \\
\hline & 50 & $1.2742 \mathrm{e}-05$ & 0.023788 & MLE \\
\hline & 100 & 1.0987 e- 05 & 0.023658 & MLE \\
\hline
\end{tabular}

\section{Conclusion}

Noting from all above tables that Mean Square Error values for Maximum likelihood estimator method is less than Mean Square Error values for ordinary least squares estimator method, thus we found that the best method for estimating the parameters is Maximum likelihood estimator method.

\section{References}

[1] Ali, A. H. and Al Kanani, I. H. "Classical methods to estimate the parameters of exponentiated weibull distribution,". Iraqi Academics Syndicate International Conference for Pure and Applied Sciences, Journal of Physics: Conference Series 2021; 1818 012078, doi:10.1088/1742$6596 / 1818 / 1 / 012078$

[2] Jalil, M. and Al Kanani, I. H. " Some estimation methods for new mixture distribution with simulation and application," 2019 IOP conference, series: materials science and engineering 571(2019) 012014

[3] Kalt, H G. and Al Kanani, I. H. " A comparison of MLE method and OLS for the estimation of modified Weibull distribution parameters by using the simulation ," ARPN Journal of Engineering and Applied Sciences, 2018; sept 13(17), ISSN 1819-6608.

[4] Nadarajah, S. "On the moments of the modified Weibull distribution," Reliability Engineering and System Safety, Elsevier, 2005; vol. 90(1), pages 114-117.

[5] Peng, W. Zhang, H. Huang, H. Gong, Z. and Liu,Y. "Satellite reliability modeling with modified Weibull extension distribution," 2012 International Conference on Quality, Reliability, Risk, Maintenance, and Safety Engineering, 2012; pp. 195-199, doi: 10.1109/ICQR2MSE.2012.6246218.

[6] Xie, M. Tang, Y. and Goh, T. N. "A modified Weibull extension with bathtub-shaped failure rate function," Reliability Engineering \& System Safety, 2002; vol. 76, no. 3, pp. 279-285. 